\title{
POTENCIALIDADES E DESAFIOS NA CONSTRUÇÃO DE UM CURSO DE MEDICINA: RELATO DE EXPERIÊNCIA
}

\author{
POTENTIALITIES AND CHALLENGES OF THE CONSTRUCTION OF A \\ MEDICINE COURSE: EXPERIENCE REPORT
}

\author{
lane Franceschet Sousa', José Carlos Souza, Eliadja Raiany Freire Moura, Thamires Durans \\ Correa
}

Universidade Federal de Santa Catarina UFSC - Campus de Araranguá', Universidade Estadual de Mato Grosso do Sul - UEMS ${ }^{2}$

\begin{abstract}
This article describes the experience of the constitution of the course of medicine in the State University of Mato Grosso do Sul (UEMS). The primary focus of the work is the course management, stressing the different preparation of the students, according to the National Curricular Guidelines for the medical graduation. During the institution of the course we shall point out the potentialities provided by the interdisciplinary work of the professors, allowing the construction of several modules and scenarios of teaching and learning, including the instruction in finger alphabet, and the optional module on the local native health. During the first year of the course, we faced many challenges, mainly associated with the lack of infrastructures and the required materials. The necessity to create a new profile of physician, with the capacity to act and resolve all the problems associated with the local health overcame all the difficulties, mainly related to the education oriented toward individuals that have always been treated with inequality in a social and historical context.
\end{abstract}

Key words: Education, Medical; Undergraduate; Curriculum; Integrality in Health.

\section{Resumo}

O artigo apresenta um relato de experiência sobre a implementação do curso de Medicina da Universidade Estadual de Mato Grosso do Sul (UEMS), enfatizando aspectos que contemplam os diferenciais na formação dos estudantes, de acordo com as recomendações das Diretrizes Curriculares Nacionais para a formação médica. $\mathrm{Na}$ construção do curso, destaca-se o trabalho interdisciplinar dos docentes como uma potencialidade, o qual respaldou a construção dos módulos e dos cenários de ensinoaprendizagem, incluindo o ensino de Libras, bem como o módulo eletivo no sistema de saúde indígena local. Muitos desafios foram encontrados durante a trajetória do primeiro ano do curso, sobretudo relacionados à falta de condições na infraestrutura e nos recursos materiais necessários. Dessa forma, a necessidade de formar um novo perfil de médico voltado para atuar na integralidade e na resolutividade de problemas locais de saúde sobrepuja todos os percalços, principalmente no que tange a uma formação voltada para os grupos de indivíduos tratados desigualmente num contexto sócio-histórico.

Palavras chave: Educação de Graduação em Medicina; Currículo; Integralidade em Saúde. 
O Curso de Medicina da Universidade Estadual de Mato Grosso do Sul (UEMS) foi concebido em 2014, na tentativa de melhorar as condições de saúde da população sul-matogrossense, cuja dispersão populacional resulta em inúmeros distritos e municípios com menos de 10.000 habitantes, onde raramente se registra a presença de profissionais médicos ${ }^{1}$.

A criação desse curso está sobejamente amparada pela Lei no 12.871 , de 22 de outubro de 2013, que instituiu o "Programa Mais Médicos" (PMM), bem como na distribuição demográfica de Mato Grosso do Sul e suas peculiaridades ${ }^{2}$. A razão médico/mil habitantes em Mato Grosso do Sul é de 1,85 , sendo que Campo Grande concentra 3,48 médicos/mil habitantes ${ }^{3}$.

Outra dimensão da realidade estadual a ser considerada é a presença da segunda maior população indígena aldeada do país, requerendo atenção específica para a saúde indígena, ainda incipiente nos três cursos de medicina existentes no Estado ${ }^{1}$.

Aqui, cabe registrar que, a partir das políticas de afirmação racial empreendidas pelo governo federal, cresceu significativamente a autodeclaração de indígenas nos diversos censos do Instituto Brasileiro de Geografia e Estatística (IBGE), razão pela qual se deu um expressivo crescimento dessa população desaldeada em diversas unidades da Federação ${ }^{4}$. Além disso, a população indígena exibe indicadores de saúde bem inferiores aos da população geral, fato que aponta para os enormes desafios que a saúde indígena coloca para os gestores ${ }^{5}$.

O curso foi criado a partir de um projeto pedagógico que privilegia as metodologias ativas de ensino-aprendizagem, em consonância com as Diretrizes Curriculares Nacionais e o $\mathrm{PMM}^{2}$. O Projeto Pedagógico do curso apresenta compromisso com a formação de um profissional com profundos vínculos regionais $\mathrm{e}$ responsabilidade social, levando em conta a rede de relacionamentos envolvida no desenvolvimento do ensino médico de qualidade, baseado na comunidade e nas necessidades regionais $^{1,6}$.

Assim, pretende-se relatar a experiência da implantação do curso de Medicina da UEMS, sob a ótica da gestão vivenciada pelos docentes, evidenciando as peculiaridades do curso para atender as demandas regionais e de populações vulneráveis.

\section{Metodologia}

O relato de experiência foi a metodologia escolhida, pois busca compreender determinada situação através da análise realizada pelos pesquisadores, podendo este interferir ou não durante $\mathrm{o}$ estudo. Este método permite relacionar a prática com a teoria, sem generalizar a primeira, nem distorcer a segunda ${ }^{7}$.

O tema deste relato de experiência foi a descrição da construção do curso de Medicina da UEMS, na Unidade Universitária de Campo Grande/MS, que iniciou em 2015. Portanto, o período incluído neste relato foi o primeiro ano de implantação do curso. Destacam-se as potencialidades e dificuldades vivenciadas durante o processo de criação dos módulos, sob o olhar da gestão do curso, bem como as peculiaridades regionais que tornam o curso singular, como saúde indígena e o estudo de libras.

\section{Resultados e Discussões}

O curso de Medicina da UEMS foi concebido a partir de quatro pilares fundamentais: (1) uso de metodologias ativas, entre elas a aprendizagem baseada em problemas e a problematização, na qual a educação modifica sua forma "bancária", assumindo outras perspectivas; (2) educação orientada para a comunidade, cujo enfoque é desenvolver atividades comprometidas com a saúde para todos, através das relações entre a universidade, os serviços de saúde e a comunidade; (3) diversificação de cenários de aprendizagem, com a vivência de situações reais nos serviços de saúde, viabilizando um profícuo diálogo entre teoria e prática; (4) educação centrada no acadêmico, o qual deve ter total responsabilidade pelo seu auto aprendizado, apoiado pelo professor ${ }^{8}$.

O curso iniciou a primeira turma em março de 2015, com 48 ingressantes, sendo que destes, 15 acadêmicos ingressaram por meio das cotas (cinco indígenas e dez negros). A construção do primeiro ano do curso ocorreu de janeiro a novembro de 2015 pela própria equipe de docentes, com apoio gerencial, técnico e pedagógico de consultoria externa. A equipe contou com 17 docentes temporários e seis docentes efetivos da instituição, totalizando 23 professores das áreas de Medicina, Enfermagem, Psicologia, Fonoaudiologia, Biologia, Farmácia e Química. Destes, 14 eram doutores, oito mestres e um especialista. Essa variedade de formação 
nas áreas básicas foi fundamental para que o caráter interdisciplinar nos módulos fosse valorizado. Todos os docentes realizaram um curso de capacitação sobre "Metodologias Ativas de Aprendizagem (PBL) e construção de módulos interdisciplinares", onde foram trabalhados temas pertinentes à educação médica como currículo integrado, estrutura curricular, interação básico-clínica em módulos temáticos, construção de problemas, construção de módulos, mapas conceituais, avaliação formativa e somativa, entre outros.

Quanto a infraestrutura, o curso conta com uma sede construída e adaptada para o modelo pedagógico do curso de Medicina. Materiais permanentes e de consumo, como equipamentos de laboratório e livros didáticos foram, aos poucos, sendo adquiridos. Algumas dificuldades comuns quando se inicia um curso novo foram enfrentadas, principalmente relacionadas aos elevados custos com equipamentos e materiais e a morosidade das instituições públicas.

A abordagem utilizada na construção do curso obedece às recomendações das novas Diretrizes Curriculares Nacionais (DCN) para a formação do médico, instituída na Resolução no 3 , de 20 de junho de $2014^{8}$. De acordo com as DCN para a formação médica, haja vista a necessária articulação entre conhecimentos, habilidades e atitudes requeridas do egresso, a formação do graduando em Medicina da UEMS foi desdobrada nas seguintes áreas: I - Atenção à Saúde; II - Gestão em Saúde; e III - Educação em Saúde $^{8}$. Para contemplar as três dimensões da formação médica, o curso foi construído a partir de uma estrutura central composta por módulos temáticos, que abrangem os quatro anos iniciais da formação, cuja integração ocorre tanto no sentido do conteúdo básico para o clínico, quanto no nível de complexidade crescente, ano após ano. A metodologia utilizada nos módulos temáticos consiste na aprendizagem baseada em problemas, cujas sessões tutoriais estão organizadas em aberturas e fechamentos dos mesmos. A semana padrão possui duas sessões tutoriais, acompanhada de um período de estudo autodirigido para cada uma delas.

A Aprendizagem Baseada em Problemas tem como base a teoria racionalista de conhecimento, segundo a qual o conhecimento dos fatos é primariamente um produto da capacidade de pensamento e, portanto, de dedução. Segundo os racionalistas, o sistema cognitivo humano procura estabelecer coerência na aquisição de conhecimentos e, ao entrar em contato com algo novo, ativa conhecimentos prévios, elabora e organiza, dentro de um contexto ${ }^{9}$.

Para dar suporte teórico-prático aos módulos temáticos, diversos conteúdos foram integrados no denominado Suporte de Aprendizagem Multidisciplinar (SAM), como anatomia, histologia, morfofisiologia e análises clínicas. Esses conteúdos são abordados através de aulas práticas ou teórico-práticas, de forma integrada entre si e ao módulo temático, as quais também devem se adequar metodologicamente a concepção didático-filosófica do curso. Acompanhando os módulos temáticos, foram construídos os módulos longitudinais, com previsão anual, compondo os seguintes cenários: Habilidades Médicas (HM); Interação Ensino, Serviço e Comunidade (IESC); Habilidades de Comunicação, Liderança e Gestão (HCLG); Iniciação e Fundamentos Científicos (IFC).

Destaca-se o módulo de HCLG, concebido de forma inovadora dentro da organização de currículos médicos nacionais, assumindo a responsabilidade de priorizar as competências relacionadas à gestão, comunicação e liderança em saúde. Os tópicos desse módulo abrangem questões de tomada de decisão em saúde, liderança, gestão em saúde e trabalho em equipe, comunicação ao paciente e na equipe, escuta, relação estudante-paciente, médicopaciente e comunicação não-verbal. Dentre os tópicos, o estudo da Linguagem Brasileira de Sinais (LIBRAS) é contemplado desde o primeiro ano do curso. O curso prevê a formação de egressos aptos a utilizar a LIBRAS com seus pacientes e equipes de saúde, contemplando a recomendação das DCN no artigo 23 e em conformidade às normas institucionais, que enfatizam a importância de se desenvolver este poderoso mecanismo de inclusão ${ }^{8}$.

No Brasil, existem mais de 2 milhões de deficientes auditivos. Tais pessoas, além serem alvo de preconceito, ainda não são incluídos totalmente nas ações de saúde, na maioria dos municípios do país. Isso porque os serviços de saúde não são adequados ao atendimento específico destes pacientes, incluindo os profissionais médicos. Muitos pacientes com deficiência auditiva conseguem obter orientações quando estão em atendimento médico apenas se tiverem um intérprete, o que nem sempre ocorre. Atualmente, a inclusão da LIBRAS nos cursos de Medicina é incipiente, porém recomendada nas $\mathrm{DCN}^{8}$. Dessa forma, a UEMS incluiu LIBRAS como conteúdo obrigatório do primeiro ao quarto ano do curso, pretendendo que seus egressos sejam fluentes na linguagem de sinais. 
Outro diferencial do Curso de Medicina da UEMS é o Módulo Eletivo no primeiro e terceiro ano, cuja finalidade é a de propiciar a inserção dos acadêmicos na conjuntura das comunidades indígenas, cumprindo com os preceitos do Projeto Pedagógico do curso, que visam alcançar uma formação médica voltada para as demandas regionais. São módulos intensivos com duração de duas semanas letivas (carga horária total de 80 horas), distribuídos em duas semanas consecutivas, ao término do primeiro semestre letivo de cada ano.

O intuito do Eletivo em saúde indígena é viabilizar aos acadêmicos uma vivência teóricoprática dos cenários e da conjuntura das comunidades indígenas, visando contribuir para uma formação de profissionais com olhares e habilidades diferenciados. Sendo assim, a introdução dessa vivência, na universidade, permite aos graduandos de Medicina um aprendizado ativo, o que aumenta o vínculo e a responsabilidade com os usuários do sistema e estimula a participação ativa junto ao SUS (e seus subsistemas) e comunidades.

No modelo contemporâneo do ensino médico no Brasil, vivencia-se a proximidade entre academia e SUS, cuja integração se mostra necessária ao aprendizado responsivo às DCN, articulando graduandos de Medicina, docentes e profissionais do SUS para um objetivo maior: melhorar o cuidado do paciente e suas especificidades, com vistas à integralidade.

Sob a ótica da gestão, o trabalho interdisciplinar durante a construção do primeiro ano do curso na UEMS ocorreu com muitos oportunidades e desafios. A lógica do trabalho interdisciplinar exige a quebra de barreiras entre as pessoas, a postura de humildade perante 0 avanço do conhecimento, aceitando que o saber da sua especialidade é limitado e não sobrepuja o saber do outro. As impressões observadas durante os trabalhos de construção dos módulos ratificaram o verdadeiro sentido de trabalho em equipe, onde a soma das competências individuais resultou em um trabalho de construção dinâmico e prazeroso.

Muitas dificuldades foram encontradas no início da implantação deste curso, cenário que geralmente se repete quando novos cursos são criados, sobretudo em se tratando de instituições públicas, cujos recursos geralmente são escassos e a morosidade faz-se presente. Outro ponto que pode ser destacado, entre os desafios vivenciados, foram as dificuldades para o desempenho dos módulos de apoio teóricopráticos, como o Suporte de Aprendizagem Multidisciplinar (SAM) e Habilidades Médicas
(HM), tanto em relação à falta de equipamentos e materiais de consumo para as aulas práticas, quanto em relação à dificuldade na adequação de métodos ativos para estes cenários.

Para que o curso se consolide com qualidade, é fundamental obter apoio político e da gestão local, já que a implementação de um novo curso de Medicina neste formato exige um aporte orçamentário considerável. Em contrapartida, os resultados futuros superam as dificuldades iniciais, tendo em vista a formação de profissionais preparados para atuar em cenários marginalizados.

Os módulos do curso foram construídos pela equipe de docentes, visando a integração curricular. A formação multiprofissional da equipe de professores tornou rico o conteúdo dos módulos, privilegiando o trabalho interprofissional e ampliando os aspectos sociais, ambientais, psicológicos e culturais. Alguns módulos, como HCLG, evidenciam o caráter inovador deste curso de Medicina, incluindo o ensino de Libras, as habilidades de gestão e liderança de equipes, aspectos fundamentais para o trabalho do médico com vistas à inclusão social.

Entretanto, alguns pontos merecem ser pontuados em relação à operacionalização dos módulos, como a execução do primeiro ano concomitante com a construção dos módulos do segundo ano, o que sobrecarregou a equipe de professores e gestores. Outro módulo que pode ser citado é o eletivo, pois apesar de ser planejado para inserir os estudantes nas comunidades indígenas, pode contar com imprevistos. Em 2015, ocorreram disputas entre os indígenas e os proprietários rurais no Mato Grosso do Sul, tornando o ambiente inseguro para a entrada dos estudantes nas aldeias fora da capital. Dessa forma, o estágio eletivo na saúde indígena do primeiro ano foi realizado nas respectivas instâncias localizadas na capital.

Especialmente pelo fato do Estado de Mato Grosso do Sul contar com uma numerosa população indígena, o incentivo para uma formação médica voltada para a resolutividade dos problemas locais de saúde é prioridade. Atualmente, a legislação vem atuando no sentido inclusivo das populações vulneráveis, como os indígenas, deficientes auditivos, negros e quilombolas, os quais até então, eram marginalizados e desconsiderados pelas políticas públicas, inclusive no âmbito do SUS.

Reitera-se, portanto, o desenvolvimento de políticas públicas de saúde que visam contribuir para a formação de médicos voltados para a integralidade como condição sine qua non para 
que as populações vulneráveis sejam atendidas em suas necessidades de saúde. Assim, pautada nas recomendações nacionais e nas políticas públicas vigentes, a UEMS tem buscado oportunizar aos acadêmicos do curso de Medicina situações de aprendizagem que contemplem o atendimento das necessidades de saúde de populações vulneráveis de forma permanente.

\section{Conclusões}

As novas diretrizes para a formação de médicos apontam para uma formação mais abrangente e com foco nas demandas regionais, premissas que foram contempladas no novo curso de Medicina da UEMS. Além disso, a formação dos estudantes incluiu o respeito a diversidade étnico-racial, linguístico-cultural e competências para o atendimento integral de pessoas com deficiência. Estes diferenciais puderam ser incorporados nos módulos de ensino devido, sobretudo, ao potencial da equipe de docentes, que trabalhou de forma interdisciplinar e interprofissional. O apoio da gestão da Universidade também foi fundamental para o sucesso na implantação do primeiro ano do curso.

Todavia, diversos obstáculos foram encontrados, como problemas de infraestrutura e falta de equipamentos no início do curso, bem como alguns imprevistos na operacionalização dos módulos práticos. O apoio da gestão local, bem como o trabalho da equipe de professores foi fundamental para superar os obstáculos encontrados. A singularidade de aspectos locoregionais dão ao curso um caráter inovador, onde se busca formar um profissional apto ao atendimento integral da população, respeitando grupos de indivíduos desvalorizados historicamente.

\section{Referências}

1. Projeto Pedagógico do Curso de Medicina (2014). Universidade Estadual do Mato Grosso do Sul. Campo Grande, MS. 2014.

2. Brasil. Lei no 12.871, de 22 de Outubro de 2013. Institui o Programa Mais Médicos, altera a Lei $n$ o 8.745, de 9 de dezembro de 1993 e no 6.932 , de 7 de julho de 1981, e dá outras providências.

3. Scheffer $M$, coordenador. Demografia Médica no Brasil 2015. Departamento de
Medicina Preventiva, Faculdade de Medicina da USP. Conselho Regional de Medicina do Estado de São Paulo. Conselho Federal de Medicina. São Paulo; 2015: 48-52.

4. Brasil. Sistema de Informação da Atenção à Saúde Indígena. População Indígena por Região, Estado, Região de Saúde e Município. Brasília, DF: Ministério da Saúde; 2013.

5. Brasil. Sistema de Informação da Atenção à Saúde Indígena. Pirâmide Populacional e etnia por distrito. Brasília, DF: Ministério da Saúde; 2013.

6. Brasil. Secretaria de Educação Superior. Proposta de expansão de vagas do ensino médico nas Instituições Federais de Ensino Superior. Brasília, DF: Ministério da Educação; 2013.

7. Léo CCC, Gonçalves A. Modalidades metodológicas em pesquisa científica, a partir de recortes da experiência de saúde coletiva, epidemiologia e atividade física da UNICAMP. Rev Educ Física 2010 jul; 21 (3): 411-41.

8. Brasil. Ministério da Educação. Resolução no 3, de 20 de junho de 2014. Institui Diretrizes Curriculares Nacionais do Curso de Graduação em Medicina e dá outras providências.

9. Tibério IFL, Atta JA, Lichtenstein A. O aprendizado baseado em problemas - PBL. Rev Med (São Paulo) 2003 jan-dez; 82(1-4): 78-80.

\section{Endereço para Correspondência}

Universidade Estadual de Mato Grosso do Sul UEMS $^{2}$

Av Dom Antonio Barbosa (MS-080), 4.155

Campo Grande - MS

CEP.: 79115-898

e-mail: camila_zanesco@hotmail.com

Recebido em 23/09/2017

Aprovado em 15/02/2018

Publicado em 11/05/2018 